\title{
émulations
}

\section{Faire œuvre de soi. Comparaison ethnographique des effets du néolibéralisme dans un mouvement spirituel et au sein d'un cercle d'affaires bruxellois}

\author{
Bénédicte Fontaine et Justine Vleminckx
}

Émulations - Revue de sciences sociales

2021, Varia, En ligne.

\section{Article disponible à l'adresse suivante}

https://ojs.uclouvain.be/index.php/emulations/article/view/Fontaine_Vleminckx

\section{Pour citer cet article}

Bénédicte Fontaine et Justine Vleminckx, « Faire œuvre de soi. Comparaison ethnographique des effets du néolibéralisme dans un mouvement spirituel et au sein d'un cercle d'affaires bruxellois », Émulations, Varia, En ligne. Mise en ligne le 13 décembre 2021. DOI : 10.14428/emulations.varia.035

Distribution électronique : Université catholique de Louvain (Belgique) : ojs.uclouvain.be

(c) Cet article est mis à disposition selon les termes de la Licence Creative Commons Attribution, Pas d'Utilisation Commerciale 4.0 International. http://creativecommons.org/licenses/by-nc/4.0/

Éditeur : Émulations - Revue de sciences sociales / Presses universitaires de Louvain https://ojs.uclouvain.be/index.php/emulations

ISSN électronique : 1784-5734

PUL PRESSES

UNIVERSITAIRES

DE LOUVAIN 


\title{
Faire œuvre de soi \\ Comparaison ethnographique des effets du néolibéralisme dans un mouvement spirituel et au sein d'un cercle d'affaires bruxellois
}

\author{
Bénédicte Fontaine ${ }^{1}$ et Justine Vleminckx ${ }^{2}$
}

\begin{abstract}
[Résumé] : Cet article, écrit à quatre mains, s'interroge sur les formes et les rôles que prennent les collectifs au sein des sociétés néolibérales, à partir d'une analyse comparée de deux recherches ethnographiques différentes: l'une menée par Bénédicte Fontaine au sein d'un cercle d'affaires bruxellois, le Club, et l'autre conduite par Justine Vleminckx au sein d'un mouvement spirituel international, la Rainbow Family. Nous proposons, à partir de nos deux terrains de recherche distincts, de donner à voir les expressions situées de l'idéologie néolibérale, et spécifiquement de l'injonction contemporaine à être entrepreneur de soi, c'est-à-dire à être manifestement un sujet actif de sa vie et de son propre bonheur. Nous nous intéresserons aux discours particuliers que cette injonction génère et les conséquences qu'elle a sur les individus, leur rapport au monde et leur rapport au collectif.
\end{abstract}

Mots-clefs : anthropologie ; néolibéralisme ; entrepreneur ; élite ; développement personnel

[Abstract]: This article, written by four hands, examines the forms and roles of collectives in neoliberal societies, from a comparative analysis of two different ethnographic studies: one conducted by Bénédicte Fontaine in a Brussels business circle, the Club, and the other conducted by Justine Vleminckx in an international spiritual movement, the Rainbow Family. Based on our two distinct and different research fields, we propose to show the situated expressions of neoliberal ideology, and specifically of the contemporary injunction to be a self-entrepreneur, i.e. to be an active subject of one's own life and happiness. We will look at the particular speeches it generates and the consequences it has on individuals, their relationship to the world and their relationship to the collective.

Keywords: anthropology; neoliberalism; entrepreneurship; elite; spirituality; selfdevelopment

\footnotetext{
${ }^{1}$ Université catholique de Louvain, LAAP, Belgique.

${ }^{2}$ Université catholique de Louvain, LAAP, Belgique.
} 


\section{Introduction : des individus entrepreneurs d'eux- mêmes}

Les avènements de Margaret Tatcher et de Ronald Reagan marquent le début d'une nouvelle ère du capitalisme (Duménil, Lévy, 2006) et de la gouvernementalité, appelée néolibéralisme, où «le meilleur moyen d'œuvrer au bien-être des hommes est de désentraver la liberté d'entreprendre et les talents individuels au sein d'un cadre institutionnel caractérisé par le libre-échange, le marché libre, et de solides droits à la propriété privée » (Harvey, 2004:16). Celle-ci vise à étendre la forme économique à tous les domaines du social. Elle ne se définit pas par la "fin du politique», mais par un nouveau programme politique caractérisé par des techniques de gouvernement ${ }^{3}$ indirectes (Foucault, 2004), par l'intermédiaire de « techniques de soi » « qui, au nom de la liberté et de l'autonomie, poussent les individus, acteurs et sujets de pouvoir, à agir sur eux-mêmes et surtout à produire du savoir sur eux-mêmes pour se constituer en sujets gouvernables» (Marquis, 2014: 22). Cette stratégie vise à rendre les sujets individuels « responsables »; elle mène «à un transfert de responsabilité dans le domaine de la responsabilité du sujet et transforme ladite responsabilité en un problème de "souci de soi" » (Lemke, 2004: 22). Les problèmes sociaux, tels que le chômage ou la maladie, sont réduits à la seule responsabilité individuelle; les réussites sont imputées au mérite personnel. Se joue ici une mutation des sujets en individus: des individus libres, déliés les uns des autres, invités à être toujours plus autonomes (Fernandez, 2011), s'inscrivant dans le grand récit de l'« homme capable » (Ehrenberg, 2018). Celui-ci est maître de sa réussite et de ses accomplissements personnels, coupable de ses difficultés et de ses échecs. Le néolibéralisme agit comme un processus d'individualisation (Fernandez, 2011) où se mettent en scène des individus sommés de s'entreprendre eux-mêmes (Crucifix, 2019; Harvey, 2004). Comme le décrit Michel Foucault, il vise l'accomplissement de "l'homo oeconomicus», "qui est un entrepreneur et un entrepreneur de lui-même», "étant à lui-même son propre capital, étant pour luimême son propre producteur, étant pour lui-même la source de ses revenus » (Foucault, 2004: 232).

Être «entrepreneur de soi » est par ailleurs souvent perçu, y compris dans le champ des sciences sociales, comme étant essentiellement une idée managériale (Getz, 2017) ayant des conséquences importantes sur le travail organisé autour d'une concurrence généralisée et sur les travailleurs, constamment surveillés par des outils de contrôle et de récompense (Le Lay, Rolo, 2017). L'injonction à être entrepreneur de soi et ses conséquences ne peuvent cependant pas être réduites au seul monde du

\footnotetext{
3 Ici le mot gouvernement est à comprendre au sens large donné par Foucault : "par gouvernement, j'entends l'ensemble des institutions et pratiques à travers lesquelles on guide les hommes depuis l'administration jusqu'à l'éducation. C'est cet ensemble de procédures, de techniques, de méthodes qui garantissent le guidage des hommes les uns par les autres qui me semble aujourd'hui en crise » (Foucault, cité par Lemke, 2004 : 18).
} 
travail et de l'entreprise. «Si on a pu parler de "subjectivation neólibeŕale", c'est que la transition néolibérale déborde la simple transformation des politiques économiques, pour donner naissance a une entière "forme de vie", une nouvelle "condition" ou le "soi entrepreneurial" deviendrait "un programme de gouvernement" » (Paltrinieri, Nicoli, 2017: 2). L'idéologie néolibérale telle que décrite par Foucault fait advenir une conception du travailleur qui devient par lui-même et pour lui-même sa propre entreprise. Cet «individu-entreprise » ne reçoit pas un salaire qui compenserait l'usage de sa force de travail, mais un revenu qui est fonction de ses compétences ; il est devenu son propre capital, en termes de savoirs, de savoir-faire, mais aussi de " pouvoir-faire». Foucault ajoute: « il faut considerer la consommation comme une activite d'entreprise par laquelle l'individu, a partir prećisement d'un certain capital dont il dispose, va produire quelque chose qui va être sa propre satisfaction " (Foucault, 2004: 232) ; plus précisément, cet individu entrepreneur de lui-même «se produi[t] comme activite vivante, comme individu-entreprise et donc comme travailleur performant, dans le travail comme dans le sport, dans l'activite ludique ou encore dans l'activite ártistique, par lesquelles on cherche a 's'eṕanouir soi-même et on apprend a concurrencer les autres » (Gorz, cité par Paltrinieri, Nicoli, 2017: 4).

Ces processus d'individualisation ont pour conséquence «le dećlin du collectif, l'eŕosion des solidariteś et l'exposition a la violence de l'ećonomie libeŕale » (Fernandez, 2011:340) : «le contrat libeŕal scelle l'ećhange de la liberte politique (disparue avec les leǵitimiteś et les transferts de pouvoir ećonomiques) contre la liberte priveé » (ibid.: 343). Ce déclin prend la forme, d'une part, de la disqualification partielle des processus de domination que sont entre autres le genre, la race, la classe, le handicap, etc., dans l'évaluation des inégalités et des injustices, réduites à des défaillances individuelles. D'autre part, ces discours entraînent un affaiblissement des organisations de lutte pour la justice sociale. Sans cesse suspectées de former des groupes de pression qui pourraient empêcher les formes spontanées du marché d'émerger, celles-ci sont démantelées, entre autres pour étouffer toute contestation sociale, aussi bien au niveau des entreprises, où le néomanagement crée des formes de travail invivable, qu'au niveau de l'État, où ces associations sont suspectées de vouloir porter atteinte aux libertés individuelles par des régulations malvenues (Harvey, 2004 ; Salmon, 2015; Wacquant, 2012).

Pour autant, le néolibéralisme ne se diffuse pas de manière uniforme, toujours identique à lui-même partout sur la planète, telle une tache d'huile (Harvey, 2004). Il est nécessaire de le penser à travers les pratiques, les discours et les résistances qu'il génère et qui se construisent autour de son idéologie. Celles-ci sont elles-mêmes situées dans le temps et dans l'espace, relatives aux groupes sociaux qui les portent et les diffusent (Crucifix, 2019). Cet article, écrit à quatre mains, s'interroge précisément sur les formes et les rôles que prennent les collectifs au sein des sociétés néolibérales, à partir d'une analyse comparée de deux recherches ethnographiques différentes : l'une menée par Bénédicte Fontaine au sein d'un cercle d'affaires bruxellois, le Club, et 
l'autre conduite par Justine Vleminckx au sein d'un mouvement spirituel international, la Rainbow Family. Nos recherches s'inscrivent respectivement dans le champ de l'anthropologie féministe du capitalisme (Bear et al., 2015) et dans le champ de l'anthropologie des nouveaux mouvements religieux (Barker, 1989; Hervieu-Léger, 2010). Menées dans le cadre de nos doctorats, elles sont fondées sur les méthodes inductives classiques en anthropologie (Laurent, 2019; Malinowski 1963; Olivier de Sardan, 2008) : le terrain ethnographique de temps long, l'observation participante et l'entretien qualitatif. La comparaison émerge dans un deuxième temps, par la prise de conscience de sujets qui nous sont communs, mais qui sont situés dans nos terrains et leurs spécificités.

Nous ${ }^{4}$ proposons, à partir de nos deux terrains de recherche distincts, de donner à voir les expressions situées de l'idéologie néolibérale, les discours particuliers qu'elle génère et les conséquences qu'elle a sur les individus, leur rapport au monde et leur rapport au collectif. Après avoir exposé les enjeux théoriques et méthodologiques de la comparaison de nos deux travaux ethnographiques, ces derniers seront exposés en trois temps: une présentation détaillée de chacun des terrains, ainsi que des outils mobilisés à la récolte de données, un exposé de la forme située de l'injonction à s'entreprendre et, enfin, une description de la manière singulière que prend le collectif dans chaque cadre. Nous conclurons enfin en déployant la comparaison, pour penser l'individualisation néolibérale en pratique, y compris dans ses contradictions.

\section{Le néolibéralisme en paroles et en actes : l'intérêt heuristique de l'ethnographie et de la comparaison}

«Les données produites par l'observation participante constituent l'un des rares accès in situ aux représentations et aux pratiques des acteurs, à leurs préoccupations, à leurs compréhensions du monde, à leur manière de le transformer " (Hilgers, 2013: 101). Dans le cadre d'une étude des impacts du néolibéralisme, l'ethnographie et ses méthodes apparaissent pertinentes pour saisir les manières dont les individus sont concrètement impactés par ce mode de gouvernementalité dans leur rapport à soi, au monde et aux groupes sociaux auxquels ils appartiennent. Au cœur de nos recherches respectives, fondées sur une série d'« observations en situation » (Ibid.), nous avons en effet pu examiner comment se joue et s'actualise, en contexte, au sein de collectifs résolument distincts, l'individualisme néolibéral dans l'ensemble des discours et des

\footnotetext{
${ }_{4}^{4}$ Le «nous », ici, et tout au long de l'article, ne renvoie pas au « nous » scientifique. II s'agit d'un « nous » pratique, qui nous situe toutes les deux comme sujets de l'énonciation et qui annonce une prise de parole commune. Les «je », quant à eux, renverront successivement à Justine Vleminckx ou à Bénédicte Fontaine. Nous nous situerons clairement en début de partie.
} 
pratiques normalisés parmi nos informateurs. Spécifiquement, nos immersions nous ont permis d'analyser des modes d'appartenance inédits, construits autour d'une aspiration commune à être entrepreneur de soi (que ce soit en termes de réussite professionnelle ou d'épanouissement personnel) et d'en révéler les modalités, le sens et les opportunités qu'ils représentent pour les personnes concernées ainsi que les tensions et les négociations constantes qu'ils impliquent.

Pour cet article, notre démarche épistémologique consiste à produire du sens autour de nos données respectives, en nous extrayant des seules réalités locales étudiées dans le cadre de nos enquêtes de terrain - les multiples lieux du Club et de la Rainbow Family - pour les faire exister ensemble et les resituer dans des logiques plus vastes qui les dépassent et les façonnent. Cette démarche comparative, construite $a$ posteriori (Geoffray et al., 2012) est née de plusieurs échanges menés sur nos travaux respectifs, à partir desquels nous avons dressé le constat d'une base conceptuelle commune à nos deux terrains: l'idéal de l'homme capable de s'entreprendre comme véritable pilier de l'idéologie néolibérale à l'œuvre.

À condition qu'elle n'élude pas les singularités de chacun des contextes étudiés (Geoffray et al., 2012) et qu'elle évite le piège de la «décontextualisation qui extrait arbitrairement les faits sociaux des contingences dont ils sont pourtant indissociables » (Bensa, cité par Hilgers, $2013: 98)$, cette mise en dialogue de terrains distincts comporte, à plus d'un titre, un intérêt heuristique. Sans être réductible à une simple généralisation de cas particuliers, la comparaison consiste à « trouver l'universel, ou plus modestement le général, c'est-à-dire des traits communs à une classe d'objets, dans le particulier» (Hilgers, 2013: 108). Dès lors qu'elle repose sur un "dénominateur commun » et qu'il ne s'agit pas d'une « comparaison pour elle-même» (Kocka, 2013), cette méthode interprétative donne du relief aux données issues de terrains singuliers en faisant émerger, de la mise en résonance de leurs réalités propres, des récurrences potentiellement révélatrices de phénomènes structurant le rapport à soi et aux autres dans nos sociétés.

\section{Deux terrains de recherche}

\section{1. « Welcome home, brothers and sisters » : la Rainbow Family}

Je (Justine Vleminckx) mène une recherche ethnographique sur la Rainbow Family en Europe $^{5}$ depuis janvier 2017 et plus spécifiquement sur les rencontres du mouvement.

\footnotetext{
${ }^{5}$ Ce choix, davantage épistémologique que méthodologique se justifie entre autres, par l'intérêt de comprendre comment se joue, dans le cadre des rassemblements européens (continentaux et locaux), l'identification intentionnelle (non héritée) à une famille universelle mythique, née dans le contexte spécifique de la révolution culturelle étasunienne des années 1960-1970.
} 
J'ai ainsi pris part aux derniers European Rainbow gatherings, en Italie dans les Dolomites Frioulanes (2017), en Pologne dans les Carpates (2018) et en Laponie suédoise (2019) ainsi qu'à plusieurs autres rassemblements organisés par la Famille belge. J'ai également participé à des événements périphériques auxquels mes interlocuteurs m'ont conviée, parmi lesquels: un festival de "rencontre spirituelle», un autre de musique psytrance ou encore une séance de " danse libre », axée sur le développement de soi par le mouvement. Je mène par ailleurs de nombreuses observations passives des groupes Facebook et des sites Internet dédiés à la Rainbow Family, considérant ces espaces numériques comme des fenêtres ouvertes sur les imaginaires, sur les idéaux et sur les pratiques communément partagés par les membres de la Famille. Ces temps d'observation sont complétés par l'analyse de documents d'archives produits par la Rainbow Family étasunienne (invitation et prospectus d'une centaine de pages diffusés à l'occasion du premier rassemblement en 1972, vidéos des fondateurs du mouvement, etc.), et par la réalisation d'une quinzaine d'entretiens compréhensifs, menés sous la forme de récit de vie centré sur le rapport des interviewés à la Rainbow Family.

La Rainbow Family est née en 1972 à l'occasion d'un rassemblement dans le Colorado (États-Unis), ayant réuni plus de 20000 personnes ${ }^{6}$. Cette rencontre a été initiée par des jeunes étudiants engagés dans des groupes pacifistes de la contre-culture américaine des années 1960-1970, connus pour leur opposition à la guerre du Vietnam (1964 - 1973), mais aussi, plus largement, pour leur résistance face aux valeurs de l'American way of life (le puritanisme sexuel et la consommation de masse, entre autres) (Beck, 2017; Macdonald, 2019; Niman, 1997). Près de 50 ans après cette première édition, la Rainbow Family a pris une ampleur considérable, lui valant d'être qualifiée de "plus importante communauté utopique nord-américaine ( (Macdonald, 2019) dont l'une des spécificités, selon l'anthropologue Adam Berger, est d'être à l'origine de rencontres New Age parmi les plus grandes de la planète (Berger, 2006: 97). Elle prend désormais la forme d'une communauté transnationale, fédérée autour de ses «Rainbow gatherings » organisés plusieurs fois par an à travers le monde pour une durée limitée, variable d'un contexte culturel à l'autre7. En fonction de leur échelle (nationale, continentale et internationale), ces rassemblements réunissent quelques centaines de personnes lorsqu'ils se déroulent au niveau local (en Belgique, par exemple), entre 1000 et 5000 participants dans le cas des rencontres européennes et jusqu'à plusieurs dizaines de milliers pour les Rainbow gatherings étasuniens.

Organisées de manière ponctuelle, et circonscrites dans un espace et un temps précis, les rencontres Rainbow consistent à expérimenter durant un mois (en Europe)

${ }^{6}$ II n'y a pas une histoire officielle et centralisée du mouvement. Selon ses adeptes, il n'y a que des « hipstories », des récits individuels d'expérience des rassemblements Rainbow.

${ }_{7}^{7}$ En Europe, la Famille Arc-en-ciel se réunit chaque année (depuis 1983) pendant un mois, selon le calendrier lunaire (d'une nouvelle lune à l'autre). Contrairement aux États-Unis, par exemple, où ils sont historiquement organisés autour de la date d'indépendance, le 4 juillet, et ne durent que sept jours. 
une vie communautaire autogérée dont l'organisation repose sur la seule base des responsabilités et des volontés individuelles. Les lieux dans lesquels les rassemblements prennent place répondent à une série de critères qui vont de la situation isolée du territoire choisi, de son caractère "préservé» de toute activité humaine à l'accessibilité à une source pour boire et cuisiner, ainsi qu'à une rivière ou un lac pour se baigner et se laver. Le temps, notion particulièrement élastique et relative pour les Rainbow, y est investi par chacun des participants selon son "feeling» ou son "énergie», mais tourne principalement autour de trois activités principales: la réalisation des tâches collectives (selon les volontés individuelles); la prise des deux repas quotidiens, précédés d'un rituel au cours duquel est célébrée l'unité qui lie inconditionnellement les membres de la Famille entre eux et les connecte au Cosmos ; et enfin, principalement l'après-midi, l'organisation de workshops proposés gratuitement et librement par les participants. Ces derniers sont dédiés à la transmission de techniques corporelles dites «thérapeutiques» et de savoirs essentiellement centrés sur l'exploration de ses potentialités (artistiques, spirituelles, physiques ou psychologiques), mais aussi, sur la découverte de la nature environnante et de ses potentiels thérapeutiques. Par l'immersion dans la Nature qu'ils proposent et l'échange de pratiques centrées sur l'épanouissement de soi, ainsi que par l'organisation d'un mode de vie libertaire sur lequel ils reposent, les rassemblements Rainbow donnent, pour mes interlocuteurs, la possibilité de dépasser leurs propres limites et de se reconnecter avec leur nature intérieure et simultanément, avec le cosmos (Vleminckx, 2020).

Par ailleurs, l'une des particularités majeures de la Rainbow Family est son ouverture inconditionnelle. Aucune obligation d'adhésion ni cotisation ne sont exigées pour intégrer le mouvement et prendre part à ses rassemblements. À l'arrivée en Rainbow gathering, chacun devient automatiquement le frère ou la sœur de l'autre et une proximité de fait, "inconditionnelle» et "authentique», se joue dès les premières interactions. Ce principe d'accueil illimité s'inscrit dans les valeurs fondamentales du mouvement qui combinent simultanément la valorisation intransigeante de la liberté individuelle et le respect fondamental d'autrui et de ses spécificités. Il est aussi rendu possible en raison des caractères expérientiel et événementiel (Salzbrunn, 2014) de la communauté arc-en-ciel : elle repose essentiellement sur l'organisation ponctuelle de rassemblements, limités dans le temps et "non territorialisés de manière permanente " (Macdonald, 2019: 223). Par le caractère temporaire de ses rencontres, la Rainbow Family évite, en principe, l'instauration de rapports de force ou l'installation de tensions sérieuses et durables autour de la gestion du quotidien ou de certains comportements jugés incompatibles avec l'esprit Rainbow. Chaque rassemblement est un nouveau départ.

En dépit de cette valeur d'ouverture, les rassemblements Rainbow réunissent des personnes au profil sociologique relativement uniforme, principalement issues de la classe moyenne éduquée, occupant une activité professionnelle dans des domaines 
particulièrement variés 8 . Sur mes terrains en Europe, je rencontre également, dans une proportion relativement importante, des personnes sans emploi par choix (selon le récit qu'ils produisent), vivant de ressources financières diverses : allocations étatiques pour les uns, revenus locatifs pour les autres ou encore, pour certains, héritages familiaux ou épargnes accumulées dans le cadre d'un ancien emploi particulièrement rémunérateur. Ce profil prédominant-classe moyenne éduquée, avec ou sans emploi est particulièrement spécifique aux rassemblements européens, dans lesquels l'interdiction de consommer de la viande, de la drogue et de l'alcool devient, de fait, un critère discriminant (au sens large). À l'inverse, dans les American Rainbow gatherings, la tolérance relative accordée à l'usage de ces produits (des espaces sont dédiés à leur consommation) contribue à attirer des personnes plus marginalisées, vagabonds, consommateurs assidus de drogues et d'alcool, attirées par le mode de vie libertaire des rassemblements et l'économie solidaire sur laquelle ils reposent (Niman, 1997).

À côté des caractéristiques socio-économiques prépondérantes des adeptes des Rainbow gatherings, il convient également de noter la présence équivalente d'hommes et de femmes, de tous âges, majoritairement valides, blancs et hétérosexuels. Cette homogénéité apparente peut s'expliquer à travers différentes hypothèses parmi lesquelles on retrouve: les difficultés d'accès aux rassemblements pour les personnes à mobilité réduite, dans la mesure où ils sont traditionnellement organisés dans des lieux reculés qui demandent, pour y parvenir, plusieurs heures de randonnée ; l'identification toujours opérante et la filiation revendiquée de la Rainbow Family au mouvement hippie des années 1960-1970 historiquement composé en priorité de jeunes issus de la classe moyenne blanche (Creagh, 2009; Hall, 1968); ou encore, tout simplement, l'impensée et l'absence de réflexion collective autour de cette homogénéité au sein des rassemblements du fait d'une conviction communément partagée selon laquelle tous les individus sont intrinsèquement dotés du même pouvoir d'agir et de choisir.

\section{2. « Vous êtes ici chez vous » : le Club}

I'ai (Bénédicte Fontaine) mené une enquête de terrain au sein d'un cercle d'affaires bruxellois, le Club9. Je suis devenue membre du Club en janvier 2019 et j'ai à partir de ce moment participé en tant que membre et avec les autres adhérents aux activités proposées. Cette entrée m’a été accordée en me faisant recommander auprès de la directrice, en mobilisant un réseau que je m'étais constitué. Au fur et à mesure de mon terrain, j'ai compris que cette place m'avait été accordée à plusieurs titres : parce que la

8 Ces observations émanent aussi bien de mes recherches sur la Rainbow Family en Europe que des analyses réalisées par Michael Niman (1997) et Charles Macdonald (2019) sur le mouvement aux États-Unis.

${ }^{9}$ Le Club a fait faillite en mai 2020 ; mon enquête de terrain, brutalement interrompue par la situation sanitaire, n'a jamais pu reprendre. 
directrice, à titre personnel, trouvait ma recherche intéressante, parce que, à titre professionnel, elle ne pouvait pas ne pas accéder à la demande de la personne qui m'avait recommandée et parce que j'incarnais en tant que femme, en tant que jeune et en tant que chercheuse plusieurs des enjeux de la politique de recrutement qu'elle menait. Enfin, cette place m’a aussi été accordée en vertu des équivalences de statut à l'œuvre: en tant que doctorante, je fais partie, aux yeux des élites économiques qui participent à la vie du cercle d'affaires, des élites universitaires. J'y ai mené une enquête en observation participante, en assistant et en participant à la vie du Club, c'est-à-dire aux évènements organisés par le Club pour ses membres (conférences, panels, discussions, apéritifs, soirées, visites organisées, compétitions de tir et de golf). Ces différents évènements m'ont permis à la fois de partager, par immersion corporelle (Andrieu, 2011), les interactions des membres entre eux, de participer aux conversations informelles et de récolter les discours officiels tenus par des orateurs et les réactions que ceux-ci produisent. J'ai complété ce travail de terrain par une trentaine d'entretiens avec certains membres, à leur domicile et sur leur lieu de travail puis par téléphone, sur Zoom ou sur Skype, par la lecture quotidienne de la presse économique belge et en consultant les biographies des hommes d'affaires présentés en référence, les ouvrages d'économie et de management qu'ils ont écrits ou qu'ils m'ont recommandés.

Le Club a été fondé à la fin des années 1990 par un homme d'affaires qui a saisi l'opportunité du déclin des cercles traditionnels pour fonder un cercle d'affaires de haut standing, bilingue et mixte. Le nombre de membres était alors limité à mille; il fallait pour devenir membre prouver sa "qualité ${ }^{10}$ », par son statut professionnel, le chiffre d'affaires de son entreprise, ses relations et son éducation. Même si le Club reste, par sa réputation, un cercle d'affaires prestigieux, ses stratégies de recrutement ont dû être révisées ces dix dernières années en raison de difficultés financières. Le Club reste essentiellement fréquenté par les élites économiques belges : des CEO (Chief Executive Officer), fondateurs, gestionnaires, administrateurs ou hauts cadres de grandes et moyennes entreprises belges ou internationales, des banquiers, des gestionnaires de fortune, des avocats et des chasseurs de têtes. Mais à ces membres traditionnels s'ajoutent désormais des start-uppers et des jeunes cadres, qui considèrent les membres comme autant de potentiels clients à démarcher, d'investisseurs à séduire ou de coachs qui pourraient les aider à faire décoller leur carrière. Pour autant, le Club est fréquenté par une population relativement homogène: malgré la mixité revendiquée, les membres sont très majoritairement des hommes (au moins $75 \%$ ), blancs, ayant entre 30 et 60 ans.

Le Club est, de plusieurs manières, le lieu d'une mise en scène du raffinement et de la distinction des classes socio-économiques supérieures (Bourdieu, 1979). Le bâtiment

\footnotetext{
10 La « qualité » des membres est un terme du terrain, et un des grands débats qui l'anime: vaut-il mieux un plus grand nombre de membres, ou moins de membres mais des membres dit « de qualité »?
} 
est un palais, spacieux et luxueux, bien en vue sur une des artères les plus fréquentées de Bruxelles, dont l'accès est limité tant de manière explicite que symbolique (Pinçon, Pinçon-Charlot, 2007). Les salles intérieures sont hautes et les plafonds ornés de moulures, de grands miroirs au cadre doré surmontent les cheminées, des antiquités meublent et décorent chaque pièce. À chaque évènement, une hôtesse accueille les membres par leur nom; des serveurs en livrée rouge remplissent les verres et débarrassent la vaisselle sale. Le Club offre ou prétend offrir un service sur mesure et personnalisé, qui témoigne de son positionnement comme lieu haut de gamme (Cousin, Lambert, 2019). Les membres eux-mêmes incarnent par leur tenue - leurs vêtements et leurs manières (Goffman,1973) - la réputation de ce lieu. Enfin, les interactions sont polies, il n'y a pas d'éclats de voix ni d'emportements. Le Club met en scène des échanges lisses, voire polissés qui se déroulent selon des codes toujours identiques. L'observation de ce milieu social s'inscrit dans la suite d'une anthropologie de la platitude telle que décrite par Marc Abélès, dont l’un des enjeux est de révéler les évidences (Abélès, 1989).

Les membres ne vivent pas au Club; ils ne s'y retrouvent qu'à l'occasion d'évènements et, si la fréquentation du Club est faite d'habitudes, elle n'est jamais pour autant le partage d'un quotidien. Ces évènements sont autant d'opportunités pour les membres de se mettre en réseau, de rencontrer des personnes intéressantes pour leur activité et leur entreprise, d'entrer en contact avec des personnes importantes et, souvent, d'entretenir et de renforcer ces liens professionnels et corporatistes. Il est très rare que se nouent au Club des accords commerciaux; de nombreux membres n'y ont même jamais mené d'affaires, ce qui est habituel à ce genre de lieux (Cousin, Chauvin, 2010). Bien qu'il soit qualifié de cercle d'affaires, le Club est davantage le lieu d'un ensemble de discours sur les entreprises, leurs objectifs et leur gestion, sur l'économie belge, européenne et mondiale et sur le management. Ces discours prennent la forme de discussions informelles entre les membres et de conférences: des allocutions d'hommes politiques orientées vers le monde économique, des discours d'intentions de grands CEO, ou des échanges d'expériences, relatant la création d'une entreprise et les stratégies de management qui ont permis son succès.

\section{Incarnations situées de l'injonction néolibérale à être entrepreneur de soi}

\subsection{S'éveiller à ses potentialités : discipline et travail permanent sur soi chez les Rainbow}

La grande majorité des personnes qui prennent part aux rassemblements Rainbow européens sont inscrits dans une démarche de développement personnel qui prend la forme d'un travail permanent sur son intériorité (Marquis, 2014) et s'intègre plus largement dans des récits croyants de type New Age (de la Torre, 2011; Ferreux, 2000; 
Heelas, 1996). La Famille Arc-en-ciel repose sur cette même conviction qu'observe Paul Heelas au sein des mouvements New Age, selon laquelle : "We malfunction because we have been indoctrinated - or, in the New Age sense of the term, been "brainwashed" by mainstream society and culture. This indoctrination obscures and cripples the power of the "real” Self » (Heelas, 1996:18-19). Pour mes interlocuteurs, il est en effet convenu que chaque individu possède en lui des potentialités illimitées pour agir sur lui-même et sur le monde, à travers la référence à des entités du type "pouvoirs créateurs » ou "puissance de l'esprit ${ }^{11}$ », comme en témoigne cet extrait d'un entretien réalisé avec Bart, l'un des initiateurs des premiers rassemblements Rainbow belges :

C'est ça qu'on apprend fondamentalement quand on devient conscient, que ce

n'est que toi-même, que tu es le seul créateur de ta vie; que ce que tu vois à l'extérieur c'est une projection de tes propres pensées. Nous sommes tous des parties de Dieu qui sommes en train de créer le monde matériel. Il faut travailler à la prise de conscience que tout part de toi-même. Il faut se libérer surtout des choses indésirables, des programmations, des peurs. (Bart, notes de terrain, 19 septembre $2017^{12}$ )

Le propos de Bart est intéressant dans la mesure où il rend compte du rapport à soi dominant au sein des rassemblements Rainbow au moins à deux niveaux. Premièrement, l'individu est «le seul créateur de [sa] vie»: tout ce qui advient est une manifestation de son intériorité, qu'il apparaît dès lors primordial d'écouter, au risque de le payer cher (Marquis, 2014: 156-157). La maladie est, par exemple, perçue comme une matérialisation dans le corps d'une mauvaise écoute et gestion de ses émotions, et donc comme une probable dissonance avec ce que son moi profond attend. Ce qui nous amène au second aspect soulevé par Bart: la «prise de conscience que tout part de toi » exige de fournir des efforts et un travail sur soi, sous la forme d'une attention permanente portée à son corps et à ses émotions, dont les multiples manifestations sont des indicateurs à prendre au sérieux pour jauger de l'authenticité de ses choix et de leur adéquation avec sa vraie nature. Cette hygiène de vie implique la conscientisation et le dépassement des émotions limitantes (la peur, le plus souvent) qui, quotidiennement, empêchent la réalisation de soi ; et, simultanément, la pratique d'une pensée positive comme clé de l'épanouissement personnel (Cabanas, Illouz, 2018 ; Marquis, 2014).

\footnotetext{
${ }_{11}^{1}$ L'usage de ces terminologies n'est pas anodin. II rend pleinement compte de l'ancrage de la Rainbow Family dans le champ des spiritualités New Age, qui reposent précisément sur la croyance en une divinité immanente, contenue en toute chose et notamment en chaque être humain.

${ }^{12}$ Notre carnet de terrain est sans doute notre principal outil de recueil des données ; il permet de prendre note à la fois des propos tenus sur le terrain et des évènements qui s'y jouent. Les différentes citations que nous utilisons en sont issues. Nous avons fait le choix dans cet article de présenter simultanément nos données et les analyses qui y sont liées. Nos descriptions, situées, sont directement ancrées dans le cadre théorique exposé en introduction.
} 
Sur mes terrains, cet idéal d'un individu « authentique» induit paradoxalement une certaine normativité puisqu'il est conditionné à un ensemble de prescriptions qu'il s'agit de manifester à travers des manières d'être conformément libéré (Vleminckx, 2020). Au sein de la Rainbow Family, par exemple, l'apparence majoritaire des corps - musclés, minces, bronzés, dénudés et visiblement décomplexés - est valorisée comme un indicateur du choix d'un mode de vie sain, au grand air, actif, au diapason avec ses besoins essentiels. Pour Brigitte, par exemple, les Rainbow gatherings, qu'elle fréquente depuis plus de vingt ans, sont des opportunités pour amincir et tonifier son corps:

En rassemblement, on apprend ce qu'est une vie saine: on marche des kilomètres pour aller pisser ou pour aller chercher l'eau. Pour plein de gens qui ont de la cellulite, pas besoin de faire des régimes, ils viennent au Rainbow. C'est une hygiène de vie qui est Waw !! Ça c'est sûr, en Rainbow, on se muscle et on mincit. (Brigitte, notes de terrain, 28 novembre 2018)

Comme dans tout contexte religieux, la nourriture y comporte une dimension éminemment morale (Liogier, 2012: 180). Durant les rassemblements Rainbow, deux repas communautaires, végétaliens, crudivores pour certains, biologiques (en principe) et frugaux sont servis quotidiennement. Ce mode d'alimentation, sain et en quantité proportionnée, représente une nécessité quasi vitale, pour mes interlocuteurs, qui y voient une occasion de se réapproprier la conscience de leur corps et de ses besoins réels.

L'impératif à être pleinement soi engage, par ailleurs, mes interlocuteurs dans une recherche insatiable d'expériences et de pratiques émancipatrices, destinées à renouer avec leur nature intérieure et, in fine, à prendre en main leur vie, leur santé et leur bienêtre. Ils pratiquent aussi bien la méditation et le yoga qu'ils ne prennent part à des rituels néo-chamaniques (huttes de sudation, cérémonie de l'Ayahuasca, etc.); ils consultent des gourous, des médiums et des coaches de vie (via une diversité de supports) et mobilisent une série d'outils et de techniques relevant des mouvances du New Age ou de certains champs de la psychologie et du développement personnel (rebirth, tests de personnalité du type MBTI, horoscope, etc.). Ces activités sont combinées comme les pièces d'un puzzle, qui donnent à conscientiser, par les sensations ou les émotions qu'elles suscitent, des dimensions de soi jusqu'ici incomprises, sousestimées ou refoulées.

Enfin, l'apparent principe d'égalité des chances selon lequel «nous sommes tous naturellement capables» dissimule en réalité une conception méritocratique de l'épanouissement de soi: celui qui ne se donne pas les moyens de sa réalisation personnelle n'a que ce qu'il mérite (Marquis, 2014 :191). Selon cette logique, les facteurs extérieurs à l'individu, que ce soient des circonstances de vie ou des contraintes systémiques, ne sont pas considérés comme responsables de ses réussites ou de ses échecs, ou de son niveau de bien-être ou de mal-être (Cabanas, Illouz, 2018). Au sein de la Rainbow Family, cette idée est notamment véhiculée sous la forme de conseils adressés dans le cadre de relations interpersonnelles, du type: «Le vrai chemin c'est le chemin 
intérieur. Tu peux te construire toute seule un paradis si tu le veux vraiment» (Alix, note de terrain, 5 juillet 2017). Elle se joue également à travers la manière dont la Rainbow Family pense les changements sociétaux auxquels elle prétend œuvrer. Se définissant comme apolitique, elle s'appuie sur la conviction que toute révolution s'opère d'abord intérieurement, à partir d'un travail sur soi et sur son équilibre de vie, et en dépit des rapports de domination systémiques. Le propos de Nicolas, jeune Bruxellois amateur des rassemblements européens du mouvement, résume de manière éloquente cette approche particulièrement structurante des discours et des pratiques au sein des rassemblements : «Le problème vient de l'intérieur. Si tout le monde était la personne qu'il veut vraiment être, alors tous les problèmes, sociaux, les guerres, etc. seraient complètement dépassés. Il n'y a pas besoin de se battre si les gens sont bien avec eux-mêmes » (Nicolas, notes de terrain, 20 mai 2017).

\subsection{La figure de l'entrepreneur : récits de réussite au sein du Club}

La figure de l'entrepreneur est constamment convoquée au Club, pour parler d'un invité prestigieux ou pour donner une couleur particulière à un évènement. J'ai longtemps cru qu'elle trouvait son sens et sa valeur dans un rapport binaire où s'opposeraient l'entrepreneur et le CEO (Chief Executive Officer, c'est-à-dire le directeur général), les réduisant l'un comme l'autre à un statut légal: l'entrepreneur est celui qui «entreprend», celui qui crée son entreprise et développe une stratégie économique et managériale qui permet sa croissance; le CEO est celui qui arrive à son poste à la suite d'un parcours d'excellence, endossant la responsabilité de faire prospérer l'entreprise dont il a la charge. Les données que je récoltais sur le terrain allaient dans ce sens : «l'entrepreneur, c'est celui qui risque son argent, le CEO, c'est celui qui mise l'argent des autres» (Laurent Dervaux, notes de terrain, 9 avril 2019); «moi, je cadre mieux avec le Club : je suis un vrai entrepreneur. J'ai créé 17 entreprises que je suis en train de fusionner en une seule » (Benoît Holvoet, notes de terrain, 23 avril 2019) ; « l'entrepreneur, c'est celui qui est prêt à tout risquer pour son idée. Tout le monde n'est pas prêt à ça » (Xavier Deconinck, notes de terrain, 29 mai 2019). Cette figure n'était pas seulement omniprésente, elle s'accompagnait aussi d'un imaginaire qui faisait des entrepreneurs qui avaient «réussi »- ici des «start-uppers »- dont l'entreprise était devenue rentable et prospère-ceux qu'il fallait admirer. Les CEO, eux, méritaient une autre forme de respect: celui dû à ceux qui exercent le pouvoir. Ils étaient, dans ce système binaire, de bons gestionnaires, aussi bien des entreprises qu'ils présidaient que de leurs études puis de leur carrière. Et leur statut sanctionnait leurs compétences et leur intelligence.

Un soir de juin, lors d'un Young Entrepreneur Afterwork, notre hôte m’interpelle violemment. Il me demande qui je suis et ce que je fais là. Il est membre du conseil d'administration du Club; il me prend pour une journaliste et craint que je ne sois là 
pour en salir davantage la réputation ${ }^{13}$. Je tente - vainement - de lui expliquer que je suis anthropologue, et que je travaille surtout «sur l'entrepreneur comme figure de réussite et sur le CEO comme figure concurrente ». Il lâche, exaspéré: « on ne peut pas être un bon CEO si on n'est pas un entrepreneur» (Jean-Marie Calleeuw, notes de terrain, 4 juin 2019). Mon erreur me saute aux yeux : l'entrepreneur n'est pas qu'un statut légal. Au Club, cette figure rend compte à la fois d'un statut, d'un idéal à atteindre, d'une figure de réussite, d'une caractéristique personnelle, d'une qualité individuelle et d'une stratégie de management.

Dans les biographies des grands patrons (Clark, 2016; Knight, 2017; Ries, 2011, par exemple), lors de l'introduction d'un orateur ou dans mes entretiens, les récits racontant les parcours individuels suivent une trame identique. Ils commencent par détailler une idée-importer du matériel informatique dans les années 1980, ouvrir des espaces de co-working, créer un service de traiteur africain -, une ambition-continuer l'entreprise familiale, travailler dans la finance, révolutionner les "process» des entreprises -, ou un intérêt dévorant pour une spécialité - les moteurs de voiture, la mise en réseau des personnes entre elles, les stratégies marketing, les nouvelles techniques de management, etc. Ce récit met en scène différents registres de la volonté individuelle: la prise de risques, l'acquisition de compétences, un investissement dans le travail qui ne «compte pas ses heures » et la mobilisation de toutes les ressources disponibles, financières et sociales. Vient ensuite, immanquablement, le récit d'un échec: « les études c'était pas fait pour moi », «ça n’a pas marché, je n’avais plus rien » ou «j'étais riche en dettes». L'emphase mise sur cette étape n'est pas anodine. Elle permet d'insister davantage sur le fait que la réussite finale-immanquable, puisque sans elle mon interlocuteur n'aurait pas sa place ni comme membre du Club, ni comme référence en son sein - est de son seul fait. Celle-ci est sanctionnée par le succès de l'entreprise, qui dégage un chiffre d'affaires important, ou par sa valorisation à un bon prix. Elle advient grâce aux qualités de stratèges, de visionnaires et de leaders des acteurs qui y sont engagés : «nous avons fait un virage à $180^{\circ}$ », « on a finalement dû admettre que cette branche n'était plus rentable, nous l'avons mise en faillite», « il faut toujours avoir un pied dans le futur », «plusieurs idées d'avance ».

L'entrepreneur comme figure de réussite au sein du Club décrit donc une attitude à adopter vis-à-vis de soi et du monde qui nous entoure, tant au niveau privé qu'au niveau professionnel. Cette manière d'être témoigne de la capacité individuelle à saisir les opportunités qui se présentent, par la prise de risque, d'une part, et, d'autre part, par la mise en œuvre volontaire de tous les moyens nécessaires pour atteindre ses objectifs - moyens dont le Club ferait partie. Elle participe d'une vision du monde comme d'un

13 Depuis sa création, le Club est l'objet de l'attention de la presse. Les difficultés financières qu'il a rencontrées ces dernières années, ainsi que la réputation de son fondateur, en prise avec la justice pour des manipulations financières au sein d'une de ses entreprises, lui ont valu des articles peu flatteurs et ont contribué à ternir sa réputation, alimentant les craintes de ses administrateurs de voir apparaître de nouveaux articles. 
ensemble d'opportunités qu'il faudrait saisir et dont chacun pourrait, s'il est suffisamment habile, tirer profit (Harvey, 2004). Elle trouve sa place dans un monde social singulier où ce qui a le plus d'importance est la survie et la croissance des entreprises, érigées en valeurs ultimes, pour lesquelles toutes les décisions nécessaires doivent être prises, parfois même au mépris des objectifs initiaux de la société.

Il est cependant important de ne pas tomber dans les simplifications dénoncées par Sylvia Yanagisako : « il a été plus ou moins assumé que nous savons où l'intérêt de la classe bourgeoise réside - à savoir dans l'accumulation du capital, le maintien du contrôle sur les moyens de production et dans l'établissement et la reproduction de l'hégémonie politique de la bourgeoisie ${ }^{14}$ » (Yanagisako, 2000: 7). La survie des entreprises comme valeur centrale, et la qualité d'entrepreneur qui lui est relative, sont les reflets d'un ensemble de préoccupations qui dépassent la question unique du profit et de sa captation, même si elle n'en est pas exclue. Ainsi ces sociétés représentent soit des héritages familiaux, industriels ou nationaux, dont les CEO endossent, en prenant leur direction, la responsabilité de continuité, soit la concrétisation d'une idée personnelle, précieuse, dans laquelle le créateur a investi son temps, son argent et son expertise. Leur responsabilité en tant qu'employeurs vis-à-vis de leurs travailleurs, c'est-à-dire le maintien voire la création d'un maximum d'emplois, est aussi un important objet de préoccupation. Enfin, sans cynisme, ils adhèrent largement au récit du capitalisme comme progrès (Tsing, 2017), auquel ils prendraient part en dirigeant adéquatement les firmes dont ils ont la charge ou en développant de nouveaux services. Cette vision implique par exemple l'idée que la croissance et le progrès technologique sont les solutions pour répondre à la crise climatique (Hultman, 2013).

\section{Le rôle du collectif dans l'accomplissement néolibéral de soi}

\subsection{Le collectif au service du développement de soi : ambivalences de la Rainbow Family}

Pour nombreux de mes interlocuteurs, participer aux rassemblements Rainbow compte parmi les expériences les plus émancipatrices qu'ils aient eu à vivre. Nicolas parle, par exemple, des Rainbow gatherings comme d'un « endroit où on peut retirer son costume et être qui on veut, quand on veut» (Nicolas, notes de terrain, 20 mai 2017); quand Astrid, l'une des pionnières du premier rassemblement belge les conçoit comme "un cadre qui permet de se connaître au plus profond» (Astrid, notes de terrain, 19 septembre 2017). Il est régulier aussi d'entendre parler du « pouvoir de guérison » des

\footnotetext{
${ }^{14} \mathrm{Ma}$ traduction. Texte original «It has been more or less assumed that we know where bourgeois interest lie - namely, in accumulating capital, maintaining control of the means of production, and establishing and reproducing bourgeois political hegemony. »
} 
rassemblements, comme pour Brigitte, citée précédemment. La fréquentation des Rainbow gatherings l'a littéralement "sauvée», alors qu'elle vivait, depuis plusieurs décennies, avec de nombreux traumas liés notamment à des situations d'abus sexuels : "Before I met Rainbow, I didn't know it was possible to tell "I love you” and somebody tell me "I love you" without intention sexual. It was a way to save my life. I could just meet myself more.» (Brigitte, notes de terrain, 5 janvier 2020)

Cet effet libérateur est intrinsèque à l'expérience des rassemblements Rainbow. Par un ensemble de dispositifs, ils créent les conditions d'expérimentation d'un Soi libéré des entraves morales et physiques, imposées par la société dominante, qui contraignent les manières d'être et de se raconter (les «formatages", pour parler comme mes informateurs). C'est le « cadre qui permet de se connaître au plus profond " auquel fait référence Astrid, un cadre qui repose sur une série de prescriptions qui encouragent au «lâcher-prise», à la «spontanéité», ou en résumé, à l'adoption de « comportements naturels».

Les Rainbow gatherings peuvent en cela être comparés aux séances de danse collective qualifiée de "spirituelle» (la Biodanza ou la danse des cinq rythmes, par exemple), telles qu'appréhendées par Michael Houseman, Marie Mazzella di Bosco et Emmanuel Thibault (2016), comme «un dispositif [d'injonctions] qui fournit aux pratiquants un vécu ayant valeur d'autorité transcendante pour la construction volontaire d'identités individuelles » (Ibid. : § 7, 1.18-19). Ils montrent en effet combien par la mise en place d'une atmosphère visuelle, sonore et olfactive spécifique (encens, lumières tamisées, musiques aux sonorités indiennes, etc.), mais aussi, grâce aux indications du facilitateur, les participants sont conduits, par la force du cadre, à adopter une attitude, des gestes et des postures qui évoquent la spontanéité et l'authenticité qu'ils cherchent à atteindre en prenant part à ces cours. "Être "spontané" revient moins à se comporter spontanément qu'à produire une représentation conventionnellement déterminée de ce qu'“agir spontanément" est supposé être» (Ibid. : § 27, 1.3-6). Ces représentations sont incarnées dans les mouvements du corps et deviennent, au fil des participations aux séances de danse, à ce point familières qu'elles se muent en "modes de ressentir et d'agir spécifiques, reconnaissables avant tout par l'expérience kinesthésique et affective qu'elles suscitent » (Ibid. : § 27, l.13-14). Ce qui est particulièrement intéressant dans ce processus, c'est la place primordiale occupée par les partenaires de danse : ils jouent simultanément un rôle de témoins de la capacité de chacun à s'éprouver et à interagir de manière "authentique", ainsi qu'un rôle de ressources. Par leurs regards, par leurs mimiques et par leurs gestes, ils permettent aux autres de développer différentes manières d'expérimenter des interactions qui se veulent « vraies », « spontanées », etc.

À l'instar de ces séances de danse décrites par Houseman et al. (2016), les rassemblements Rainbow ont pour but annoncé d'offrir une expérience insolite, hors du quotidien, construite comme un temps et un espace de reconnexion à sa nature profonde par le retour à la Nature (Liogier, 2012) et par l'adoption d'un mode de vie qui 
se veut inspiré de traditions qualifiées d'« ancestrales », sans que cette désignation soit précisée: "The Rainbow is by nature a space of connection with the Earth, with yourself and with others. It is a healing and natural reconnection space. We apply the way of life of the tribes that still live in deep connection with nature» (extrait de l'invitation au rassemblement belge de l'été 2017).

Durant les rassemblements, plusieurs dispositifs impliquent un processus de mise en conformité qui passe par l'intériorisation de représentations incarnées de ce qu'est être «libéré », « spontané » ou "pleinement soi » (Vleminckx, 2020). Comme pour les séances de danses observées par Houseman et al. (2016), les autres y jouent en permanence un rôle de témoin et de ressource. Les workshops, organisés quotidiennement et proposés gratuitement, constituent par exemple l'un de ces dispositifs. Par l'organisation répétée de ces ateliers, il s'agit d'affirmer et d'incarner collectivement la nécessité d'une discipline de vie qui implique un travail permanent sur soi.

\subsection{Le Club comme lieu où faire classe}

Le Club est un entre-soi. Bien qu'il se revendique de toutes les élites (politiques, intellectuelles, diplomatiques), ses membres sont avant tout issus de l'élite économique et financière, c'est-à-dire des chefs et des cadres d'entreprises et de banques. Les membres sont majoritairement des hommes; ils possèdent au moins un diplôme universitaire et ont un parcours scolaire et professionnel qui a entraîné une «mobilité internationale » : ils ont étudié ou travaillé en dehors de la Belgique pendant un temps parfois long. Ils font aussi preuve d'une grande mobilité professionnelle : ils obtiennent une promotion ou sont débauchés à un rythme régulier. Ils ont majoritairement plus de 35 ans, sont mariés et ont des enfants. Une grande partie d'entre eux se décrit ellemême comme sportive. Ces activités - course à pied, cyclisme, voile, tennis, golf - tout comme leur vie de famille, font partie des informations classiques de leur présentation; elles sont des marqueurs de classe et de réussite au même titre que leur statut professionnel. Les corps, au Club, sont étonnamment similaires: les membres sont soignés, élancés et sportifs, bien coiffés et bien habillés -ils portent des costumes élégamment coupés, adaptés à la saison, des chemises aux cols amidonnés et des chaussures cirées -, ils se tiennent droits et ont des gestes mesurés.

Cet entre-soi est constaté à tous les instants par ceux qui le constituent. Ils remarquent, par exemple, " qu'ils se croisent tout le temps », « qu'ils connaissent tout le monde ici», ou: «nous venons au Club parce que nous y sommes entre nous, et que c'est agréable » (notes de terrain, 4 juin 2019). Le Club n'est pas le seul lieu où ils se retrouvent dans un entre-soi similaire, parfois composé des mêmes personnes: ils siègent dans les mêmes conseils d'administration, fréquentent les chambres de commerce, sont affiliés à un club de golf ou de tennis, etc., autant de lieux où l'homogénéité socio-économique se rejoue (Cousin, Chauvin, 2012; Pinçon-Charlot, 2007). 
Cet entre-soi permet aux membres de rencontrer et d'échanger avec des personnes qui partagent les mêmes préoccupations et, dans une certaine mesure, les mêmes enjeux face au monde contemporain - en tant que chefs d'entreprise. Le Club n'est pas un lieu où se nouent des relations commerciales ou des amitiés - un réseau professionnel peut s'y construire, ou en tout cas s'y renforcer, mais sur le très long terme. Par ailleurs, les relations entre les membres sont soumises à deux contradictions. Celle relevée par Immanuel Wallerstein: ils travaillent dans les mêmes secteurs, ils sont donc en concurrence pour les mêmes marchés (Wallerstein, 1983); et celle générée par le récit de l'«individu entrepreneur de lui-même» auquel ils adhèrent à travers la figure de l'entrepreneur, qui entraîne une perception de l'individu comme se suffisant à lui-même. Le Club, alors, remplit un rôle plus complexe, et plus diffus que la création de liens commerciaux ou d'amitié, et favorise, à travers ses contradictions les liens liquides, presque utilitaristes (Bauman, 2004). Frédéric, CEO, explique que quand on dirige une entreprise, qu'on est tout en haut, on n'a plus de collègue. On est seul. Cette solitude de chef est doublée du sentiment qu'ils ont de vivre dans un monde qui ne serait pas favorable aux patrons, aux entreprises ou à la réussite, qui empêcheraient, l'un et l'autre, de partager sereinement les préoccupations pour l'entreprise et sa gestion (notes de terrain, 26 novembre 2019). Le Club, dans une certaine mesure, permet de pallier ce sentiment de solitude. Il ne crée pas tout à fait des amis - c'est rarement le cas - mais rend possible, parfois, cette collégialité qui fait défaut. Il n'est pas un lieu à partir duquel faire collectif: c'est un lieu à partir duquel faire classe. Il joue alors un double rôle: il se fait le relais et le porte-voix d'un certain nombre de discours et de pratiques à travers lesquels les valeurs de ses membres, en tant qu'ils exercent un pouvoir important au sein du monde économique belge et de ses entreprises, sont partagées, diffusées et renforcées, d'une part, et, d'autre part, il permet à ses membres de se reconnaître entre eux, comme occupant des places et des statuts équivalents au sein de la société et comme partageant des préoccupations et des réponses similaires à celles-ci, afin, entre autres, de conserver leur place favorable au sein des différents rapports de pouvoir à l'œuvre. Dans ce cadre, la figure de l'entrepreneur, qui vient s'adosser à l'impératif de survie et de croissance des entreprises, sont des valeurs partagées autour desquelles les membres se retrouvent et qui fondent le Club.

\section{Conclusion : faire œuvre de soi sous le regard des autres}

La comparaison a posteriori que nous avons cherché à construire ici répondait à la volonté de dépasser la simple énonciation de l'injonction à «être entrepreneur de soi » comme un fait néolibéral toujours identique à lui-même et réductible aux seules logiques managériales. La Rainbow Family et le Club donnent en effet à voir des formes extrêmement différentes d'accomplissement de soi et de ce qui est considéré en leur sein comme la réussite, au sens d'une vie enviable. 
Ainsi, dans le cadre de la Rainbow Family, la référence à la figure contemporaine de l'entrepreneur n'a jamais lieu explicitement. Dans son sens strict, elle aurait plutôt tendance à être rejetée vu le regard critique et le positionnement en rupture posés par la majorité des membres de la communauté à l'égard du monde du travail, de l'entreprise en particulier et du salariat en général. En revanche, l'injonction à l'entrepreneuriat de soi comme capacité de l'individu à être manifestement un sujet actif de sa vie (Dardot, Laval, 2009) est profondément structurante du rapport à soi et aux autres construit au sein de cette communauté spirituelle. Elle prend la forme d'une discipline de vie et d'une attention constante portée à soi et à ses émotions, comme condition d'épanouissement personnel et, en prolongement, selon une approche holistique, comme conditions d'un " changement de paradigme ", selon l'expression d'usage au sein de la Rainbow Family pour désigner la réforme radicale de la société dominante à laquelle les Rainbow aspirent.

Pour les membres du Club, la réussite est quantifiable et sanctionnée par le récit d'un parcours professionnel et privé qui l'incarne. Les diplômes, d'abord, puis la montée en hiérarchie, soit au sein d'une même entreprise soit vers des entreprises de plus en plus grandes, et, une fois à la tête de ces entreprises, les chiffres d'une croissance (augmentation du nombre de travailleurs, du chiffre d'affaires, du nombre de pays où elles sont présentes), mais aussi un mariage et des enfants et une belle propriété, sont autant de preuves de la valeur du membre ou de l'orateur. Ces réussites ne sont imputables qu'à l'esprit d'entreprise de l'individu. Celui-ci étend ses compétences à mener sa vie et sa carrière à la société dans laquelle il occupe des responsabilités. Les membres sont autant d'«individus-entreprise», qui sont enjoints à se gérer comme étant leur premier capital, qu'ils doivent faire prospérer (Foucault, 2004; Paltrinieri, Nicoli, 2017). Ces récits mettent en scène des réussites toujours individuelles, que chacun ne devrait qu'à son seul mérite; ils occultent largement les privilèges initiaux dont ces individus jouissent, tel leur capital culturel, social ou financier (Bourdieu, 1979), et s'ils en prennent acte, c'est pour souligner à quel point ils ont réussi à les faire prospérer.

Ces modèles opposés correspondent tous deux à des incorporations situées de l'injonction néolibérale à être entrepreneur de soi : ils impliquent la valorisation de la responsabilité individuelle comme condition de l'accomplissement personnel. Ils ignorent l'un et l'autre d'une part les capitaux préalables dont les individus peuvent jouir et d'autre part les différents rapports de pouvoir qui traversent la société (la race, la classe, le genre, par exemple) et qui sont en leur faveur, facilitant leur accomplissement et leur réussite. Ces réussites, situées, profondément différentes et opposées dans leur trajectoire et dans leurs effets, sont racontées comme déconnectées des conditions matérielles et collectives de leur réalisation, comme le fait d'individus qui ne doivent leur accomplissement qu'à eux-mêmes et à leur travail. Celui-ci est à la fois un travail sur soi-même et la mise en œuvre individuelle, ou prétendue telle, de trajectoires scolaires puis professionnelles valorisées. La comparaison permet de mettre à jour les 
porosités à l'œuvre entre les discours rapportés ici, malgré leurs dissensions fondamentales et leurs objectifs opposés, témoignant d'un mouvement qui traverse la société et impacte à tous niveaux la perception des individus qui évoluent en son sein.

Par-delà une lecture des effets de l'injonction à « être entrepreneur de soi » sur le rapport de l'individu à lui-même et sur la production de discours qui la mettent en récit, nos deux terrains invitent aussi à porter une attention particulière sur des manières de faire collectif à partir de prescriptions néolibérales, et ce malgré l'individualisation qu'elles entraînent. Les deux groupes que nous avons mis en dialogue dans cet article permettent de penser des formes d'appartenance qui reposent sur la nécessité de s'entourer d'autres qui observent et valident le niveau de réussite ou d'épanouissement de l'individu, mais également sa capacité à sans cesse travailler à sa réalisation. Ce besoin de validation s'inscrit au plus profond de la dimension expressive de la culture de l'authenticité qui s'exerce de manière radicale au sein des sociétés consuméristes (Taylor, 2014) : les dynamiques propres à la production des identités, comme résultat de choix personnels, reposent sur des modes de reconnaissance "qui mettent constamment en jeu des formes - virtuelles ou actuelles - de communauté » (Gauthier, 2017: 100). En participant aux activités de la Rainbow Family et du Club, nos acteurs de terrains se reconnaissent donc réciproquement à travers leur appartenance au groupe et dans leur performance à incarner (Goffman, 1973) l'idéal d'entrepreneuriat de soi, transcrit au sein de ces groupes sous la forme de normes singulières et structurantes. Les collectifs observés - et leurs membres, dans un processus dynamique - produisent ainsi une idée située de ce qu'est la réussite individuelle et normalisent les moyens pour y parvenir. Ils prescrivent les bonnes manières de s'entreprendre soi-même, ainsi que les objectifs que cette entreprise de soi vise. Les individus qui s'y engagent participent à la production et à la reproduction de ces normes et deviennent autant d'instances de validation de la réussite des autres membres.

Notons enfin que les collectifs que nous avons observés se caractérisent aussi par leur liquidité (Bauman, 2004). Leurs membres n'adhèrent pas de manière figée et une fois pour toute à ces groupes. Ils vont et viennent, et appartiennent par ailleurs à d'autres collectifs qui répondent ou répondront à d'autres besoins. Le rapport à ces groupes d'appartenance est en ce sens instrumental, voire opportuniste. Les individus qui y recourent, en prenant part à leurs activités et en s'y identifiant dans une certaine mesure, cherchent à optimiser leur possibilité d'accomplissement de soi, grâce aux multiples dispositifs que le collectif leur offre et dans un cadre normatif qui définit les modalités de cet épanouissement. Ainsi le Club est approprié par ses membres comme le lieu de la construction de relations professionnelles utiles à la réussite de l'entreprise ou de la carrière et de l'échange d'informations et de pratiques (placements financiers, techniques managériales, échanges internationaux, implications de futures lois, etc.). La Rainbow Family, quant à elle, répond à la quête d'authenticité dans laquelle ses adeptes sont engagés et en définit les modalités, à partir de l'expérience que proposent 
ses rassemblements (immersion en pleine nature, ateliers centrés sur le développement de soi, valorisation de la « libération de soi », etc.). Cette forme d'appartenance au groupe, loin d'être superficielle, donne à penser comment des individus tentent aujourd'hui de répondre, dans le cadre de collectif, à l'impératif contemporain à être acteur du développement de son potentiel, de son autonomie et de sa flexibilité. Au sein du Club comme au sein de la Rainbow Family, ces injonctions néolibérales deviennent signifiantes d'une destinée et d'un idéal de vie communs, dans un contexte néolibéral profondément individualiste, marqué par de nombreuses incertitudes quant à l'avenir.

\section{Bibliographie}

ABÉLÈs M. (1989), «Pour une anthropologie de la platitude. Le politique et les sociétés modernes », Anthropologie et sociétés, vol.13, n³, p.13-24.

ANDRIEU B. (2011), Les avatars du corps. Une hybridation somatechnique, Montréal, Liber.

BARKer E. (1989), New Religious Movements: A Practical Introduction, Londres, H.M. Stationery Office.

BaUmanZ. (2004), L'amour liquide. De la fragilité des liens entre les hommes, Paris, Éditions du Rouergue.

BEAR L., Ho K., TSing A., YANAGiSAKo S. (2015), « Gens. A Feminist Manifesto for the Study of Capitalism », Society for Cultural Anthropology. En ligne, consulté le 14 octobre 2021. URL: [https://culanth.org/fieldsights/gens-a-feminist-manifesto-for-thestudy-of-capitalism].

BECK G. (2017), True Stories: Tales from the Generation of a New World Culture, Bloomington, iUniverse.

Berger A. (2006), The Rainbow Family: An Ethnography of Spiritual Postmodernism, Thèse de doctorat, University of St Andrews.

BOURdiEU P. (1979), La distinction. Critique sociale du jugement, Paris, Les Éditions de Minuit.

CABANAS E., Illouz E. (2018), Happycratie. Comment l'industrie du Bonheur a pris le contrôle de nos vies, Paris, Premier Parallèle.

CLARK D. (2016), Alibaba. L'incroyable histoire de Jack Ma, le milliardaire chinois, Paris, Éditions François Bourin.

Cousin B., CHAuvin S. (2010), « La dimension symbolique du capital social: les grands cercles et Rotary clubs de Milan », Sociétés contemporaines, vol. 77, n¹, p.111-37.

Cousin B., Chauvin S. (2012), "L'entre-soi élitaire à Saint-Barthélémy», Ethnologie française, $\mathrm{n}^{\circ}$ 42, p. 335-345.

COUSIN B., LAMBERT A. (2019), "Grandes fortunes et services personnels », Actes de la Recherche en Sciences Sociales, vol. 230, n 5, p. 4-11.

CREAGH R. (2009), Utopies américaines: Expériences libertaires du XIXe siècle à nos jours, Paris, Agone. 
CRUCIFIX C. (2019), Les argents de la fin du progrès, Thèse de doctorat, UCLouvain, Louvain-la-Neuve.

DARDOT P., LAVAL C. (2009), La nouvelle raison du monde. Essai sur la société néolibérale, Paris, La Découverte.

DE LA TORRE R. (2011), «Les rendez-vous manqués de l'anthropologie et du chamanisme », Archives de sciences sociales des religions, $n^{\circ} 153$, p. 145-158.

DUMÉNIL G., LÉVY D. (2006), «Une théorie marxiste du néolibéralisme », Actuel Marx, $\mathrm{n}^{\circ} 40$, p. 24-38.

Ehrenberg A. (2018), La mécanique des passions. Cerveau, comportement, société, Paris, Éditions Odile Jacob.

FERNANDEZ B. (2011), "Le temps de l'individuation sociale», Revue du MAUSS, n³8, p. 339-348.

FERREUX M.-J. (2000), Le New Age. Ritualités et mythologies contemporaines, Paris, L'Harmattan (" Nouvelles études anthropologiques »).

FouCAult M. (2004), Naissance de la biopolitique (1978-1979), Paris, EHESS/Gallimard/ Seuil (« Hautes études »).

GAUTHIER F.(2017), « De l'État-nation au Marché. Les transformations du religieux à l'ère de la mondialisation », Revue du MAUSS, $n^{\circ}$ 49, p. 92-114.

Geoffrey M.-L., LAPLANCHE-SERVIGNe S., LE RENARD A. (2012), "Comparer a posteriori. Retour sur une expérience collective de recherche», Terrains et Travaux, vol. 2, $\mathrm{n}^{\circ} 21, \mathrm{p} .165-180$.

GETZ I. (2017), L'entreprise libérée. Comment devenir un leader libérateur et se désintoxiquer des vieux modèles, Paris, Librairie Arthème-Fayard.

GofFman E. (1973), La mise en scène de la vie quotidienne. 1. La présentation de soi, Paris, Les Éditions de Minuit.

Hall S. (1968), The Hippie: An American "Moment", Thèse de doctorat, University of Birmingham.

HaRvey D. (2004), Une brève histoire du néolibéralisme, Paris, Les Prairies Ordinaires.

HeElas P. (1996), The New Age Movement. The Celebration of the Self and the Sacralization of Modernity, Cambridge, Blackwell.

HERVIEU-LÉGER D. (2010), « Le partage du croire religieux dans des sociétés d'individus », L’Année sociologique, vol. 60, n¹, p. 41-62. En ligne, consulté le 24 septembre 2021. URL : www.cairn.info/revue-l-annee-sociologique-2010-1-page-41.htm

HILGERS M. (2013), « Observation participante et comparaison: contribution à un usage interdisciplinaire de l'anthropologie », Anthropologie et Sociétés, vol. 37, n¹, p. 97115.

Houseman M., MAZzella Di BosCo M., Thibault E. (2016), « Renaître à soi-même », Terrain [En ligne], $n^{\circ} 66$, p. 62-85. En ligne, consulté le 24 septembre 2021. URL: http://journals.openedition.org/terrain/15974 
Hultman M. (2013), « The Making of an Environmental Hero: A History of Ecomodern Masculinity, Fuel Cells and Arnold Schwarzenegger », Environmental Humanities, vol. 2, p. 79-99.

Knight P. (2017), L'art de la victoire. Autobiographie du fondateur de Nike, Paris, Hugo Poche.

KOCKA J. (2013), « Les approches comparées face à la globalisation », Sociologie du travail, vol. $55, n^{\circ} 2$, p. $237-244$.

LAURENT P.-J. (2019), Devenir anthropologue dans le monde d'aujourd'hui, Paris, Karthala.

LE LAY S., Rolo D. (2017), «Ce que le néolibéralisme fait au travail: une étude de cas en centre d'appels téléphoniques », Terrains/Théories, $n^{\circ}$ 6, En ligne, consulté le 26 février 2020. URL : http://journals.openedition.org/teth/905

LEMKET. (2004), «"Marx sans guillemets”: Foucault, la gouvernementalité et la critique du néolibéralisme », Actuel Marx, n 36, p.13-26.

LIOGIER R. (2012), Souci de soi, conscience du monde: Vers une religion globale?, Paris, Armand Colin.

MACDONALD C. (2019), L'ordre contre l'harmonie. Anthropologie de l'anarchie, Paris, Éditions Petra, p. 201-229 ("Terrains et théories anthropologiques»).

MALINOWSKi B. (1963), Les argonautes du Pacifique occidental, Paris, Gallimard.

MARQuis N. (2014), Du bien-être au marché du malaise: La société du développement personnel, Paris, Presses universitaires de France.

Niman M. (1997), People of the Rainbow. A Nomadic Utopia, Knoxville, The University of Tennessee Press.

OlIVIER DE SARDAN J.-P. (2008), La rigueur du qualitatif. Les contraintes empiriques de l'interprétation socio-anthropologique, Louvain-la-Neuve, Bruylant- Academia.

PALTRINIERI L., Nicoli M. (2017), « Du management de soi à l'investissement sur soi. Remarques sur la subjectivité post-néo-libérale». Terrains/Théories, $\mathrm{n}^{\circ}$ 6. En ligne, consulté le 26 février 2020. URL: https://journals.openedition.org/teth/929

Pinçon M., PinçON-Charlot M. (2007), Les ghettos du gotha, Paris, Seuil.

RIES E. (2011), The Lean Start-up, London, Penguin Books.

SALmon A. (2015), "Néolibéralisme, nouveau management et plaisir», Connexions, vol. 103, n $\mathrm{n}^{\mathrm{1}}$, p. 21-38.

SALZBRUNN M. (2014), "Appartenances en fête: entre l'ordinaire et le spectaculaire», Social Compass, vol. 61, $\mathrm{n}^{\circ} 2$, p. 250-260.

TAYLOR C. (2014), « La consommation et la radicalisation de la culture moderne de l'authenticité et de l'expressivité », Revue du MAUSS, vol. 2, n² 2, p. 68-74.

TSING A. (2017), Le champignon de la fin du monde. Sur la possibilité de vivre sur les ruines du capitalisme, Paris, La Découverte.

VlEMINCKX J. (2020), « Des ambivalences dans la démarche d'élévation spirituelle au sein de la Rainbow Family, L'ethnographie, n³-4. En ligne, consulté le 14 mars 2021. URL : https://revues.mshparisnord.fr/ethnographie/index.php?id=706 
WACQUANT L. (2012), «Three Steps to a Historical Anthropology of Actually Existing Neoliberalism », Social Anthropology, vol. 20, n¹, p. 66-79.

WALLERSTEIN I. (1983), Historical Capitalism, Londres, Verso.

Yanagisako S. (2000), Producing Culture and Capitalism. Family Firm in Italy, Princeton, Princeton University Press. 